\title{
Clinical and Radiographic Predictors of Neurological Outcome Following Posterior Fossa Decompression for Spontaneous Cerebellar Hemorrhage
}

\author{
Md. Shamiul Alam Siddique ${ }^{1 *}$, Md. Mahfuzur Rahman², Rashed Mahmud ${ }^{3}$, Md. Rafiqul Islam $^{4}$, Md. Ruhul Quddus ${ }^{5}$
}

${ }^{1}$ Md. Shamiul Alam Siddique, Assistant professor of Neurosurgery, Mymensingh Medical College, Mymensingh, Bangladesh

${ }^{2}$ Md. Mahfuzur Rahman, Assistant Professor of Neurosurgery, Dhaka Medical College, Dhaka, Bangladesh

${ }^{3}$ Rashed Mahmud, Assistant Professor of Neurosurgery, Dhaka Medical college, Dhaka, Bangladesh

${ }^{4}$ Md. Rafiqul Islam, Associate Professor of Neurosurgery, Dhaka Medical College, Dhaka, Bangladesh

${ }^{5}$ Md. Ruhul Quddus, Medical Officer Department of Neurosurgery, Bangabandhu Sheikh Mujib Medical University (BSMMU), Dhaka, Bangladesh

DOI: $10.36347 /$ sasjm.2021.v07i03.008 $\quad$ | Received: 06.03.2021 | Accepted: 24.03 .2021 | Published: 28.03 .2021

*Corresponding author: Dr. Md. Shamiul Alam Siddique

\section{Abstract}

Background: Cerebellar haemorrhages occur most frequently in the area of the dentate nucleus. From this site they can spread to involve most of the cerebellar hemisphere and occasionally cross the midline to involve the other side. Objective: This study was to evaluate the clinical and radiographic predictors of neurological outcome following posterior fossa decompression for spontaneous cerebellar haemorrhage. Methods: The Study was conducted in the Department of Neurosurgery, in collaboration with the Department of Anesthesiology and Radiology Imaging, Dhaka Medical College Hospital, Dhaka, Bangladesh to find out the common indications of non-contrast enhanced CT scan suggestive of spontaneous cerebellar haemorrhage. 97 cases were randomly selected for the study whose common indication of non-contrast enhanced CT scan suggestive of spontaneous cerebellar haemorrhage. Clinical examination and evaluation were done from May 2012 to December 2013. Statistical analysis of the results was obtained by using window-based computer software devised with Statistical Packages for Social Sciences (SPSS-22). Results: Table shows mean age was 62.94( \pm 8.88$)$, minimum age was 37 and maximum age was 72 years, maximum age group was 61-70 years of age 39(40.2\%). Figure shows Modified Rankin Scale (MRS) in GCS score $\leq 8$ 14(93.3\%) were in MRS G-3, 06(60\%) on MRS G-4 and 16(72.7\%) were in MRS G-6. In GCS score 9-13 15(65.2\%) were MRS G-0, 12(60\%) MRS G-1, 07(100\%) MRS G-2, 04(40\%) MRS G-4 and 04(18.2\%) MRS G-6. Conclusions: The configuration of the fourth ventricle on CT scans is a useful sign for selection of the appropriate surgical procedure, compared with estimation of the size of the hematoma. The outcomes of patients with ventricular compression and GCS scores of less than 8 at the time of treatment were poor, despite aggressive treatment.

Keywords: Radiographic Predictors, Neurological, Cerebellar haemorrhages.

Copyright $(\odot 2021$ The Author(s): This is an open-access article distributed under the terms of the Creative Commons Attribution 4.0 International License (CC BY-NC 4.0) which permits unrestricted use, distribution, and reproduction in any medium for non-commercial use provided the original author and source are credited.

\section{INTRODUCTION}

Cerebellar haemorrhage comprises 5 to $13 \%$ of all parenchymal intracranial haemorrhage. It is a serious condition with a high mortality. The diagnosis must be made early because most patients die within the first 48 hours [1]. Hypertension is the underlying cause in the majority $(60-80 \%)$ [2]. In a series of 10 adult patients with cerebellar haemorrhage, hypertension was the most common etiological factor accounting for $70 \%$ of the cases [3]. Other series have also reported, hypertension to be the commonest etiological factor [4]. Arteriovenous malformation, blood dyscrasia, trauma, neoplasm, anticoagulant and aneurysm account for the rest [5]. Certain rare aetiological factors for cerebellar haemorrhage (i.e.) amyloid angiopathy have also been reported [6]. Cerebellar haemorrhage has been reported in more than 20 cases following supratentorial craniotomy and two cases after cervical spine surgery. Obstruction of flow in the internal jugular vein at the site where the vein descends across the transverse process of the Atlas is likely cause of the venous hypertension resulting in cerebellar haemorrhage [7]. Cerebellar haemorrhages occur most frequently in the area of the dentate nucleus. From this site they can spread to involve most of the cerebellar hemisphere and occasionally cross the midline to involve the other side. Not infrequently, they extend into the cerebellar peduncles, and they commonly rupture into the fourth ventricle. Only rarely do they directly involve the brain 
Shamiul Alam Siddique et al., SAS J Med, Mar, 2021; 7(3): 104-109

stem, but the brainstem is frequently deformed by pressure from the clot [5]. In I960, emphasized some of the important features of cerebellar haemorrhage, but according to him the clinical picture was too protean and neurological signs were not specific enough to establish a clinical diagnosis without laboratory and radiologic investigations [8]. Some study insisted that the diagnosis of cerebellar haemorrhage should be made strictly on the basis of clinical criteria without resisting to time consuming radiological investigations [9]. The clinical features have been divided into early, intermediate and late stages [5].

\section{OBJECTIVES}

This study was to evaluate the clinical and radiographic predictors of neurological outcome following posterior fossa decompression for spontaneous cerebellar haemorrhage.

\section{METHODOLOGY}

The Study was conducted in the Department of Neurosurgery, in collaboration with the Department of Anesthesiology and Radiology Imaging, Dhaka Medical College Hospital, Dhaka, Bangladesh to find out the common indications of non-contrast enhanced CT scan suggestive of spontaneous cerebellar haemorrhage. 97 cases were randomly selected for the study whose common indication of non-contrast enhanced CT scan suggestive of spontaneous cerebellar haemorrhage. Clinical examination and evaluation were done from May 2012 to December 2013. Other necessary investigations were done if clinically indicated and to prepare the patient for anesthesia. Then the cases were again evaluated according to preoperative findings. Inclusion criteria was Diagnosed clinically and radiologically. Exclusion criteria were Patient with use of antithrombolytic and anticoagulant drugs, Severe comorbid disease, Age less than 10 years and Patients with cerebellar haemorrhage extending into the brainstem will excluded from the study Finally, the specimen was sent for histopathological examination and correlated with clinical and preoperative findings. Statistical analysis of the results was obtained by using window-based computer software devised with Statistical Packages for Social Sciences (SPSS-22).

\section{RESULTS}

Table shows mean age was $62.94( \pm 8.88)$, minimum age was 37 and maximum age was 72 years, maximum age group was 61-70 years of age $39(40.2 \%)$. Table shows clinical presentation in out of 36 cases where GCS score $\leq 8$, among them $28(77.78 \%$ ) were vomiting, 16(44.44\%) were dizziness and 07(19.44\%) were convulsion. Out of 42 cases where GCS score 913 , among them headache $33(78.57 \%)$, vomiting $07(16.67 \%)$, dizziness $21(50 \%)$, ataxia $30(71.43 \%)$ and dysarthria were $22(52.38 \%)$. Out of 19 cases where GCS score 14-15 among them, headache 16(84.21\%), vomiting 03(15.79\%), dizziness $10(52.63 \%)$, ataxia $11(57.89 \%)$ and dysarthria were 04(21.05\%). In about 24 patients intra-ventricular haemorrhage were more in patients with poor GCS group $\leq 8 \mathrm{GCS}$ that was $13(54.17 \%)$ and out of 73 patients intra-ventricular haemorrhage were less in higher GCS 14-15 that was $18(24.66 \%)$ ( $\mathrm{p}<0.05)$ that was statistically significant. Table shows Glasgow outcome scale Death 16(72.7\%) on admission $\leq 8 \mathrm{GCS}, 04(18.2 \%)$ in $9-13 \mathrm{GCS}$ and $02(09 \%)$ were in $14-15$ GCS $(p<0.05)$ that was statistically significant. Sever disability $06(60 \%)$ in $\leq 8$ GCS, $04(40 \%)$ on admission GCS 9-13 (p>0.05) that was not statistically significant. Moderate disability $14(53.8 \%)$ in $\leq 8 \mathrm{GCS}, 11(42.3 \%)$ in $9-13 \mathrm{GCS}$ and $01(3.8 \%)$ were in admission GCS $14-15(\mathrm{p}<0.05)$ that was statistically significant. Good recovery $23(58.9 \%)$ were in $9-13$ GCS and $16(41 \%)$ were in admission GCS 14-15. $(\mathrm{p}<0.05)$ that was statistically significant. Figure shows Modified Rankin Scale (MRS) in GCS score $\leq 8$ $14(93.3 \%)$ were in MRS G-3, 06(60\%) on MRS G-4 and $16(72.7 \%)$ were in MRS G-6. In GCS score 9-13 $15(65.2 \%)$ were MRS G-0, 12(60\%) MRS G-1, 07(100\%) MRS G-2, 04(40\%) MRS G-4 and 04(18.2\%) MRS G-6. In GCS scores 14-15 08(34.8\%) were in MRS G-0, 08(40\%) MRS G-1, 01(6.7\%) MRS G-3 and $02(9.1 \%)$ were MRS G-6 ( $\mathrm{p}<0.05)$ that was statistically significant for all grade except MRS G-4.

Table-1: Demonstrated the distribution of study patients according to age.

\begin{tabular}{|l|l|l|}
\hline Age group & $\mathbf{n = 9 7}$ & $\mathbf{\%}$ \\
\hline 31-40 years & 03 & 03.1 \\
\hline 41-50 years & 08 & 08.2 \\
\hline 51-60 years & 26 & 26.8 \\
\hline 61-70 years & 39 & 40.2 \\
\hline$>70$ years & 21 & 21.6 \\
\hline Total & 97 & 100.0 \\
\hline Mean SD & $62.94( \pm 8.88)$ & $37-72$ years \\
\hline
\end{tabular}

Table-2: Clinical presentation distribution according to GCS score

\begin{tabular}{|c|c|c|c|c|c|c|c|c|}
\hline \multirow[t]{2}{*}{ Clinical presentation } & \multicolumn{6}{|l|}{ GCS score } & \multicolumn{2}{|l|}{ Total } \\
\hline & $n=36(\leq 8)$ & $\%$ & $n=42(9-13)$ & $\%$ & $n=19(14-15)$ & $\%$ & $n=97$ & $\%$ \\
\hline Headache & 00 & 0.0 & 33 & 78.57 & 16 & 84.21 & 49 & 50.52 \\
\hline Vomiting & 28 & 77.78 & 07 & 16.67 & 03 & 15.79 & 38 & 39.18 \\
\hline Dizziness & 16 & 44.44 & 21 & 50 & 10 & 52.63 & 47 & 48.45 \\
\hline Ataxia & 00 & 0.0 & 30 & 71.43 & 11 & 57.89 & 41 & 42.27 \\
\hline Dysarthria & 00 & 0.0 & 22 & 52.38 & 04 & 21.05 & 26 & 26.80 \\
\hline Convulsion & 07 & 19.44 & 00 & 0.0 & 00 & 0.0 & 07 & 7.22 \\
\hline
\end{tabular}


Shamiul Alam Siddique et al., SAS J Med, Mar, 2021; 7(3): 104-109

Table-3: Intra-ventricular haemorrhage distribution according to GCS score

\begin{tabular}{|c|c|c|c|c|c|c|c|c|c|}
\hline \multirow[t]{3}{*}{ Intra-ventricular haemorrhage } & \multicolumn{6}{|c|}{ GCS score } & \multicolumn{2}{|l|}{ Total } & \multirow[t]{3}{*}{ P value } \\
\hline & $\leq 8$ & & $9-13$ & & 14-15 & & \multirow[t]{2}{*}{$\mathrm{n}=97$} & \multirow[t]{2}{*}{$\%$} & \\
\hline & $n=36$ & $\%$ & $n=42$ & $\%$ & $n=19$ & $\%$ & & & \\
\hline Present & 13 & 54.17 & 10 & 41.67 & 01 & 4.17 & 24 & 100.0 & \multirow[t]{3}{*}{0.04} \\
\hline Absent & 23 & 31.51 & 32 & 43.84 & 18 & 24.66 & 73 & 100.0 & \\
\hline Total & 36 & 37.11 & 42 & 43.30 & 19 & 19.59 & 97 & 100.0 & \\
\hline
\end{tabular}

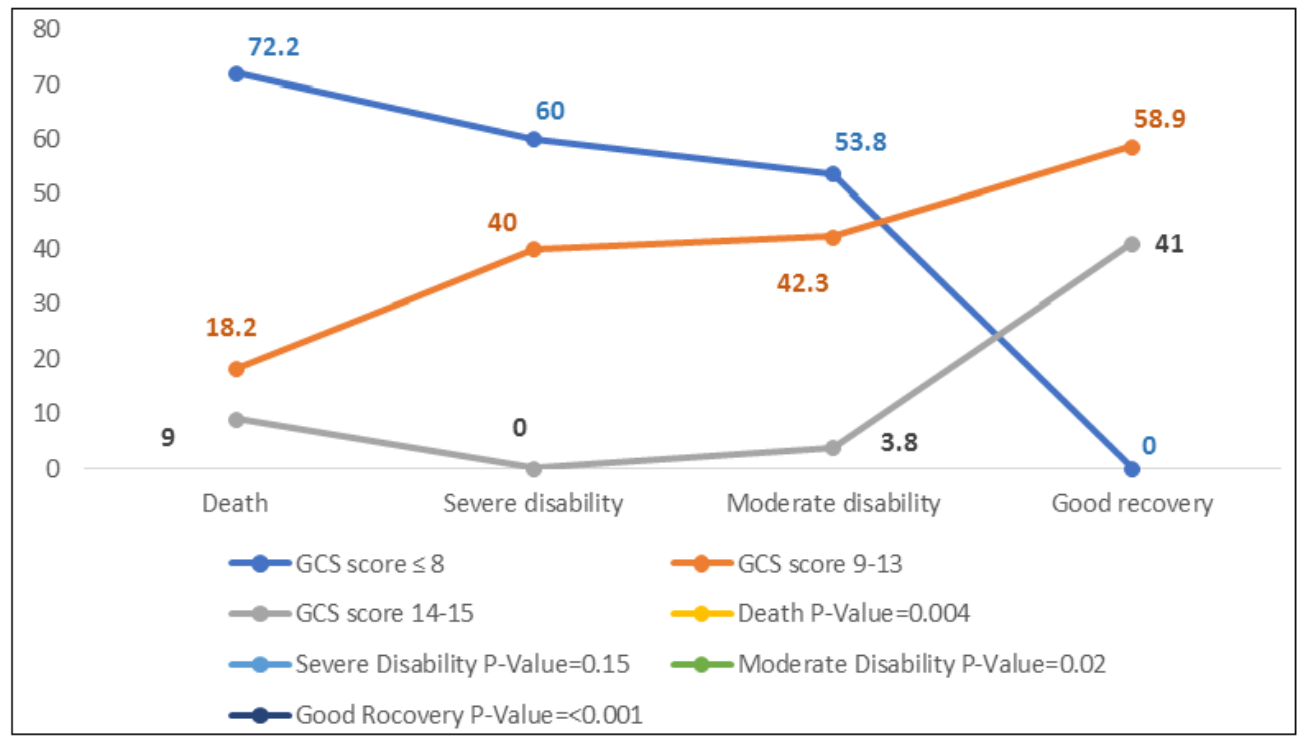

Fig-1: Glasgow outcome scale distribution according to GCS score

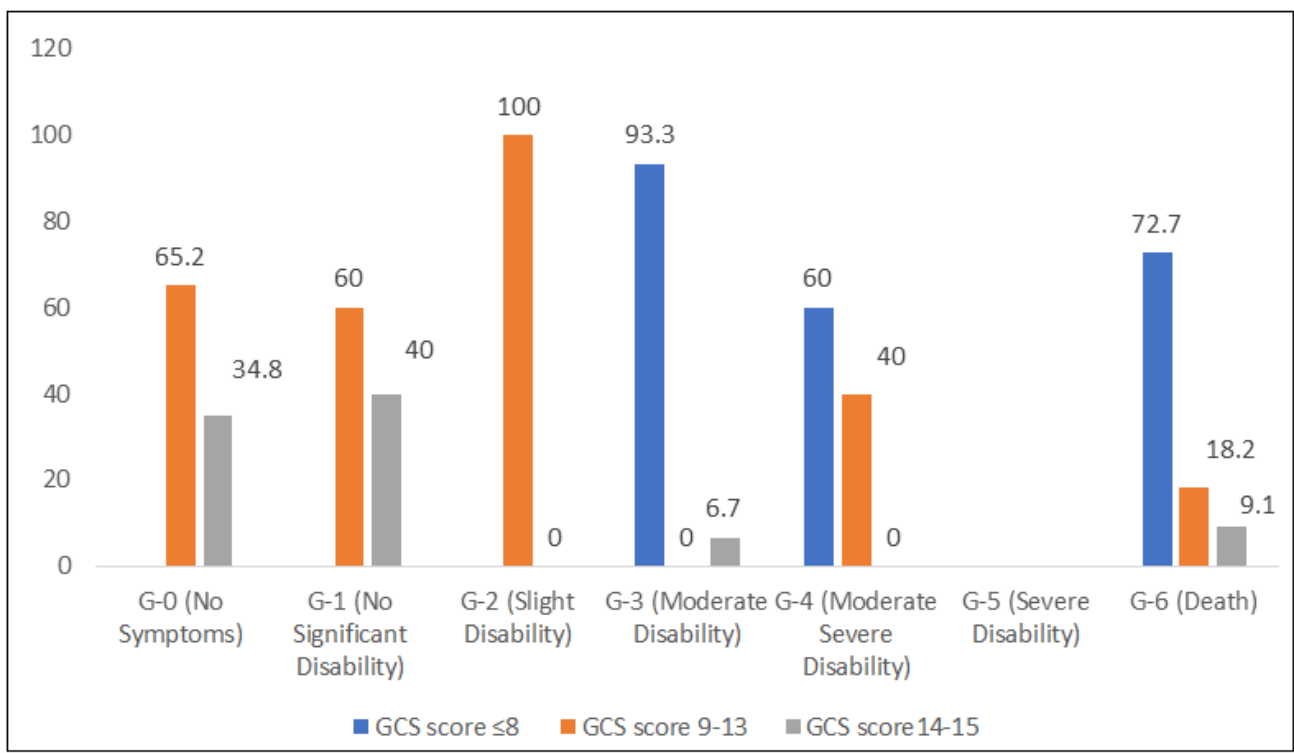

Fig-2: Modified Rankin Scale (MRS) distribution according to GCS score

\section{DISCUSSION}

Only during the past 10 years, spontaneous cerebellar haemorrhages have become a well - defined nosological entity. The surgical indication remains debatable. Our primary objective in this study is to set the criteria for undertaking surgery by determining the critical diameter of the hematoma and considering the patients' neurological status (Glasgow Coma Scale). Spontaneous cerebellar haemorrhages were admitted to the Neurosurgery depertment, Dhaka Medical College
Hospital. On admission all patients underwent a standard neurological examination, Glasgow Coma Scale and a computed tomographic scan. The diameter and the site of the hematoma, a coexisting tight posterior fossa, and the presence of hypertensive hydrocephalus were the criteria, in association with the patients' neurological status, used as indications for surgery. The aim of this paper is to describe the clinical and radiographic predictors of neurological outcome following posterior fossa decompression for 
spontaneous cerebellar haemorrhage. Patient was purposely enrolled according to the inclusion and exclusion criteria. Total 97 cases were enrolled in this study. In this study shows mean age was $62.94( \pm 8.88)$, minimum age was 37 and maximum age was 72 years, maximum age group was 61-70 years of age 39(40.2\%). Male were $56(58 \%)$ and female were $41(42 \%)$, male female ratio 1.36:1. The average age of the cohort was 71.1 years \pm 10.8 . Twenty $(54.1 \%)$ were female. Twenty-nine $(78.4 \%)$ were known to be hypertensive. In a recent study, reported the average age of their group was 64.4 years. The advanced age of the patients with $\mathrm{SCH}$ in our series agreed with the published data indicating that $\mathrm{SCH}$ occurs in the sixth to eighth decades of life. This tendency to occur in elderly persons does not necessarily lead to a poor prognosis. M. Salvati, study comprised 50 patients, 30 males $(60 \%)$ and 20 females $(40 \%)$, with an average age of 61 years. Eight patients (16\%) (2 men and 6 women; M:F ratio 1:3) were older than 80 years. No significant differences were found between the sexes.

Some studied 37 patients with cerebellar haemorrhages, 30 of whom underwent suboccipital craniectomy and the rest were treated conservatively [10]. The mean age of their cohort was 64.8 years. Comparisons were made between the two groups with the outcome measure being GOS score at discharge and three months later. They evaluated the following clinical and radiographic parameters: size of hematoma, GCS, presence of long tract signs, and hydrocephalus. The prediction of outcome by a logistic regression model included both the size of the hematoma and the treatment modality. In their study the size of the hematoma was the only significant predictor of the outcome, with larger hematomas predicting worse outcome. They suggested that surgical evacuation should be pursued in all patients presenting with large hematoma $(>3 \mathrm{~cm})$. Their study did not consider other important clinical variables including age and the presence of other comorbidities, which are potential confounding variables. Some study demonstrated that in patients with cerebellar haemorrhage requiring hematoma evacuation, the preoperative size does not correlate with either postoperative GCS score, Rankin score, or with GOS score [11]. This discrepancy between different studies illustrates that a single clinical or radiological factor is unlikely to be sufficient and that prospective RCTs will be needed to find rational treatment algorithms. Currently at least three therapeutical schemes have gained wide acceptance. One was submitted by some study [12]. This protocol uses the patient's GCS-score at admission and the haematoma size on CT-scan as discriminating factors. According to this, patients with a high GCS score at admission (14-15) and with a small haematoma $(<4 \mathrm{~cm}$ diameter) should be treated conservatively. Patients with a lower GCS score (13 or <13) and/or a large hematoma (4 $\mathrm{cm}$ or more) should undergo decompressive surgery; comatose patients whose brainstem reflexes are lost at admission do not benefit from surgical intervention. The algorithm was subsequently tested prospectively in a second group of patients $(n=49)$ with comparable results [12].

The first surgical evacuation of a cerebellar hematoma was reported in 1906 by Ballance [13]. To date this has been common practice in the treatment of cerebellar haemorrhages. Some studied 94 patients diagnosed with cerebellar haemorrhage [14]. The median age of their cohort was 74 . The overall surgical probability was $33 \%$. They demonstrated that at their institution, a hematoma size of $<3 \mathrm{~cm}$ does not influence their decision to operate on patients younger than 70 years old if clinical deterioration occurs, but surgery is withheld from patients older than 70 with large hematomas. Some study demonstrated that even though the degree of postoperative improvement was less in patients older than 70, age did not influence long term outcome reflected by Rankin score, and GOS. Some reported the results of 22 patients suffering from cerebellar haemorrhages with a mean age of 56 years $[15,16]$. The study evaluated admission GCS, maximal hematoma diameter on CT, location of the hematoma, $\mathrm{IVH}$, hydrocephalus, and the degree of quadrigeminal cistern involvement (compression, obliteration, or the presence of blood). The outcome measure was GOS at the time of discharge. Their study showed that patients with a hematoma size $3 \mathrm{~cm}$ or larger and a GCS worse than 9 had an unfavorable outcome despite surgery. Patients with hematoma sizes smaller than $3 \mathrm{~cm}$ and a GCS of 9 or better had a favorable outcome. For patients with hematomas larger than $3 \mathrm{~cm}$ and a GCS score of 9 or better, outcome depended on the location of the hematoma, and the concurrent presence of hydrocephalus, quadrigeminal cistern involvement, and IVH. The worse results were observed if hydrocephalus, quadrigeminal cistern obliteration, and IVH were present. The authors were aggressive in the treatment of patients with poor presenting GCS, however were not specific in terms of the mode of surgical therapy or cerebrospinal fluid diversion. Moreover, GOS was obtained only at the time of discharge, and major clinical criteria were not factored in their analysis including the presence of other medical comorbidities [16].

In this study out of 56 patients obstructive $\mathrm{HCP}$ were more in patients with poor GCS group $\leq 8$ GCS, and obstructive HCP less were in higher GCS group 14-15 GCS, $(\mathrm{p}<0.05)$ that was statistically significant. Besides evaluating the above-mentioned prognostic indicators some studied 31 patients with spontaneous cerebellar haemorrhage who had a low GCS score at admission and underwent MRI [17]. Obliteration of $4^{\text {th }}$ ventricle and the permesencephalic scan and the presence of areas of high signal intensity in the brainstem in T2 weighted images were studied. Brainstem was a significant prognostic factor for determining outcomes for patients with spontaneous 
cerebellar haemorrhage who were in poor grade [17]. In this study Glasgow outcome scale Death 16(72.7\%) on admission $\leq 8 \mathrm{GCS}, 04(18.2 \%)$ in $9-13 \mathrm{GCS}$ and $02(09 \%)$ were in $14-15$ GCS $(\mathrm{p}<0.05)$ that was statistically significant. Sever disability $06(60 \%)$ in $\leq 8$ GCS, 04(40\%) on admission GCS 9-13 (p>0.05) that was not statistically significant. Moderate disability $14(53.8 \%)$ in $\leq 8 \mathrm{GCS}, 11(42.3 \%)$ in $9-13 \mathrm{GCS}$ and $01(3.8 \%)$ were in admission GCS $14-15(\mathrm{p}<0.05)$ that was statistically significant. Good recovery $23(58.9 \%)$ were in 9-13 GCS and 16(41\%) were in admission GCS 14-15. $(\mathrm{p}<0.05)$ that was statistically significant. In a recent study, reported their experience in treating 57 patients with cerebellar haemorrhage with suboccipital surgical evacuation [18]. In their study, age, preoperative GCS score, hematoma size, acute hydrocephalus, tight posterior fossa, brain stem compression, fourth ventricular compression, and the presence of IVH were examined. The GOS score was the outcome measure used. The mortality rate was $25 \%$. At the last follow-up $47 \%$ of patients had a good outcome with GOS score of 4 and 5 , and $28 \%$ of patients had a poor outcome with GOS score of 2 or 3 . Preoperative GCS score was found to be a significant determinant for clinical outcome, with a lower presenting GCS score predicting a poorer outcome [18].

In this study Modified Rankin Scale (MRS) in GCS score $\leq 8$ 14(93.3\%) were in MRS G-3, 06(60\%) on MRS G-4 and 16(72.7\%) were in MRS G-6. In GCS score 9-13 15(65.2\%) were MRS G-0, 12(60\%) MRS G-1, 07(100\%) MRS G-2, 04(40\%) MRS G-4 and 04(18.2\%) MRS G-6. In GCS scores 14-15 08(34.8\%) were in MRS G-0, 08(40\%) MRS G-1, 01(6.7\%) MRS G-3 and 02(9.1\%) were MRS G-6 (p <0.05) that was statistically significant for all grade except MRS G-4. Among patients who were followed up, 17 out of 29 patients $(58.6 \%)$ lived functionally independently (Rankin 0-2), one (3.4\%) patient had a moderate disability (Rankin 3), none had a major disability or was in vegetative state (Rankin 4-5), and 11 (37.9\%) had died (Rankin 6). Using the GOS score, 18 (62.1\%) patients had a favorable outcome (lived independently or with minimal disability (GOS score 4-5), and none had a poor outcome (GOS score 2-3) [11]. Some study among patients who were followed up, 17 out of 29 patients $(58.6 \%)$ lived functionally independently (Rankin 0-2), one (3.4\%) patient had a moderate disability (Rankin 3), none had a major disability or was in vegetative state (Rankin 4-5), and 11 (37.9\%) had died (Rankin 6) [11].

The treatment algorithm by some study is described in the section "Suggested treatment algorithms". $36.8 \%$ of all surviving patients had no or only mild disability defined as GOS score 5 or mRS score 0-2 [19]. The percentage of patients with no or only mild disability was three times higher among patients that were managed medically than in the surgical group (78.7\% vs. $23.7 \%)$.
Limitations of Study

This study was not without limitation. The limitations of the studies were, conducted in only one center and the sample size was small and study period was short.

\section{CONCLUSION}

Decision-making in the management of spontaneous cerebellar hematomas requires careful consideration and interpretation of the various criteria. The configuration of the fourth ventricle on CT scans is a useful sign for selection of the appropriate surgical procedure, compared with estimation of the size of the hematoma. The outcomes of patients with ventricular compression and GCS scores of less than 8 at the time of treatment were poor, despite aggressive treatment. Nonsurgical treatment may be undertaken for this group of patients; however, apart from absent brainstem reflexes, there are no identified criteria to exclude all of these patients from aggressive treatment. Aggressive early surgical evacuation of the hematoma and ventricular drainage for patients with ventricular compression, before deterioration of the consciousness level, is emphasized. Simply, our treatment protocol uses GCS scores to select patients for surgical treatment and uses the degree of fourth ventricular compression to select the surgical procedure.

RECOMMENDATIONS: Large scale, multicentres study should be under taken.

\section{REFERENCES}

1. Mezzadri JJ, Otero JM, Ottino CA. Management of 50 spontaneous cerebellar haemorrhages. Acta neurochirurgica. 1993 Mar;122(1):39-44.

2. Mohadjer M, Eggert R, May J, Mayfrank L. CTguided stereotactic fibrinolysis of spontaneous and hypertensive cerebellar hemorrhage: long-term results. Journal of neurosurgery. 1990 Aug 1;73(2):217-22.

3. Little JR, Tubman DE, Ethier R. Cerebellar hemorrhage in adults: diagnosis by computerized tomography. Journal of neurosurgery. 1978 Apr 1;48(4):575-9.

4. Yoshida S, Sasaki M, Oka H, Gotoh O, Yamada R, Sano K. Acute hypertensive cerebellar hemorrhage with signs of lower brainstem compression. Surgical neurology. 1978 Aug $1 ; 10(2): 79-83$.

5. Heros RC. Cerebellar haemorrhage and infarction. Stroke. 1982;13:106-9.

6. Cuny E, Loiseau H, Rivel J, Vital C, Castel JP. Amyloid angiopathy-related cerebellar hemorrhage. Surgical neurology. 1996 Sep $1 ; 46(3): 235-9$.

7. Seoane E, Rhoton Jr AL. Compression of the internal jugular vein by the transverse process of the atlas as the cause of cerebellar hemorrhage 
Shamiul Alam Siddique et al., SAS J Med, Mar, 2021; 7(3): 104-109

after supratentorial craniotomy. Surgical neurology. 1999 May 1;51(5):500-5.

8. Mc.Kissock W, Richardson A, Wash L.(1960); Spontaneous cerebellar haemorrhage A Study of 34 cases treated surgically, Brain 1960; 83:1-9.

9. Fischer CM, Picard EH, Palak A, Dadal A, Acute hypertensive cerebellar haemorrhage. Diagnosis and surgical treatment. J nerrv.Ment.Dis 1965, 140: 38-57.

10. Cohen ZR, Ram Z, Knoller N, Peles E, Hadani M. Management and outcome of non-traumatic cerebellar haemorrhage. Cerebrovascular Diseases. 2002;14:207-13.

11. Dahdaleh NS, Dlouhy BJ, Viljoen SV, Capuano AW, Kung DK, Torner JC, Hasan DM, Howard 3rd MA. Clinical and radiographic predictors of neurological outcome following posterior fossa decompression for spontaneous cerebellar hemorrhage. Journal of Clinical Neuroscience. 2012 Sep 1;19(9):1236-41.

12. Kobayashi S, Sato A, Kageyama Y, Nakamura H, Watanabe Y, Yamaura A. Treatment of hypertensive cerebellar hemorrhage-surgical or conservative management?. Neurosurgery. 1994 Feb 1;34(2):246-51.

13. Ballance H. Case of traumatic haemorrhage into the left lateral lobe of the cerebellum, treated by operation, with recovery. Surg Gynecol Obstet. 1906; 3:223-5.

14. Wijdicks EF, Louis EK, Atkinson JD, Li H. Clinician's biases toward surgery in cerebellar hematomas: an analysis of decision-making in 94 patients. Cerebrovascular Diseases. 2000;10(2):93-6.

15. Dahdaleh NS, Dlouhy BJ, Viljoen SV, Capuano AW, Kung DK, Torner JC, Hasan DM, Howard 3rd MA. Clinical and radiographic predictors of neurological outcome following posterior fossa decompression for spontaneous cerebellar hemorrhage. Journal of Clinical Neuroscience. 2012 Sep 1;19(9):1236-41.

16. Luparello V, Canavero S. Treatment of hypertensive cerebellar haemorrhage-surgical or conservative management? Neurosurgery. 1995; 37:552-3.

17. Yanaka K, Meguro K, Fujita K, Narushima K, Nose T. Postoperative brainstem high intensity is correlated with poor outcomes for patients with spontaneous cerebellar hemorrhage. Neurosurgery. 1999 Dec 1;45(6):1323-8.

18. Dammann P, Asgari S, Bassiouni H, Gasser T, Panagiotopoulos V, Gizewski ER, Stolke D, Sure U, Sandalcioglu IE. Spontaneous cerebellar hemorrhage-experience with 57 surgically treated patients and review of the literature. Neurosurgical review. 2011 Jan;34(1):77-86.

19. Kobayashi S, Sato A, Kageyama Y, Nakamura H, Watanabe Y, Yamaura A. Treatment of hypertensive cerebellar haemorrhage: surgical or conservative management? Neurosurgery. 1994;34:246-51. 